Esta sección esta abierta para todos los lectores de la Revista, a la que pueden enviar sus preguntas, comentarios o críticas a los artículos que hayan sido publicados en los últimos números, teniendo en cuenta la posibilidad de que los autores aludidos puedan responder. Podrá aceptarse la comunicación de investigaciones preliminares, o de intervenciones en brotes que no hayan sido publicados ni sometidos a publicación en otra revista; así como, algunos comentarios sobre problemas de salud pública, ética y educación médica. La extensión máxima aceptable es de 1000 palabras, con un máximo de seis referencias bibliográficas (incluyendo la referencia del artículo que la motivó, cuando sea el caso) y una tabla o figura. Esta puede ser enviada a revmedex@ins.gob.pe.

\section{ESTIMACIÓN DE LA COMORBILIDAD CRÓNICA SOBRE EL COSTO SANITARIO EN PACIENTES ANCIANOS CON TRASTORNOS NEUROLÓGICOS}

\section{ESTIMATION OF CHRONIC CO-MORBIDITY ON HEALTH COSTS IN ELDERLY PATIENTS WITH NEUROLOGICAL DISORDERS}

Antoni Sicras-Mainar ${ }^{1, a}$, Ruth Navarro-Artieda ${ }^{2, b}$, Amador Ruíz-Torrejón ${ }^{3, c}$, Alexandra Prados-Torres ${ }^{4, a}$; Grupo de Estudio ACG España

Sr. Editor. El envejecimiento progresivo de la población, los crecientes avances diagnósticos y terapéuticos, junto a una mayor educación sanitaria, son algunos de los factores que ocasionan un incremento en el uso de los recursos sanitarios ${ }^{(1)}$. Es frecuente la presencia de múltiples enfermedades en personas de edad avanzada, prueba de ello es que diferentes estudios poblacionales han comprobado que el número de enfermedades aumenta con la edad (2). Por ejemplo, se ha descrito

\footnotetext{
1 Dirección de Planificación, Badalona Serveis Assistencials. Barcelona, España.

2 Hospital Germans Trías i Pujol. Barcelona, España.

3 Dirección de Gestión de Atención Primaria. Palma de Mallorca, España.

4 Instituto Aragonés de Ciencias de la Salud. Zaragoza, España.

a Médico, Doctor en Medicina, especialista en Salud Pública; ${ }^{b}$ Médico, especialista en Documentación Médica; ' Médico, especialista en Atención Primaria
}

Grupo de Estudio ACG España: Grupo de Cataluña (Concepción Violan-Fors, Alba Aguado-Jodar, Soledad Velasco-Velasco, Milagrosa Blanca-Tamayo, Esperanza Escribano-Herranz, Ferran Flor-Serra, Josep Ramón Llopart-López, Daniel Rodríguez-López, Encarna SánchezFontcuberta, Josep Maria Vilaseca-Llobet), Grupo de Baleares (José Estelrich-Bennasar, Vicente Juan Verger, María Antonia Mir-Pons, María Vega Martín-Martín, Juan Antonio Pérez-Artigues) y Grupo de Aragón (José María Abad-Díez, María Mercedes Aza Pascual-Salcedo, Daniel Bordonaba-Bosque, Amaya Calderón-Larrañaga, Francisca GonzálezRubio, Anselmo López-Cabañas, Jesús Magdalena-Belio, Beatriz Poblador-Plou, Antonio Poncel-Falcó).

Recibido: 13-07-11 Aprobado: 20-07-11 que el $24 \%$ de los mayores de 65 años tienen cuatro o más condiciones crónicas ${ }^{(3)}$. De estos, los trastornos neurológicos (TN), que comprenden enfermedades del sistema nervioso central y periférico, son muy relevantes en edades avanzadas, lo cual genera más costos, no solo para el sistema sanitario (costos directos), sino para toda la sociedad (costos indirectos) ${ }^{(4)}$, siendo frecuentemente atendidos en centros de atención primaria (AP) ${ }^{(5)}$. Por ello, realizamos un estudio para describir la asociación entre el número de comorbilidades crónicas y el costo sanitario en mayores de 64 años con presencia de algún trastorno neurológico.

Se elaboró un estudio multicéntrico empleando historias clínicas informatizadas de pacientes seguidos en $\mathrm{AP}$, provenientes de trece centros de AP de Cataluña, pertenecientes a cuatro entidades proveedoras (BadaIona Serveis Assistencials, Consorci Sanitari Integral, GesClínic y La Roca del Vallès); que tienen asignada una población aproximada de 313500 habitantes, en su mayoría de nivel socioeconómico medio-bajo, urbana y de predominio industrial. Fueron incluidos en el estudio todos los pacientes que demandaron atención y que estuvieran adscritos a los centros el 2008. Se excluyeron los sujetos trasladados a otros centros de AP y los desplazados de otras zonas.

Se analizaron las siguientes variables: a) edad y sexo; $b$ ) comorbilidad (episodios) y c) costo sanitario. La comorbilidad se obtuvo según los criterios definidos en la Clasificación Internacional de la AP (CIAP-2) ${ }^{(6)}$. El modelo de costos desarrollado para cada paciente se estableció diferenciando los costos semifijos (funcionamiento) y los variables (en función de la actividad desarrollada por cada paciente que los genera). Las principales partidas contables pertenecientes al primer grupo fueron: personal, compras, servicios externos y un conjunto de gastos pertenecientes a los servicios de estructura y gestión del centro. La asignación de los gastos semifijos a cada paciente, se hizo en función de un criterio de reparto por visita realizada. Los costos variables se calcularon en función de las solicitudes diagnósticas, prescripción farmacéutica o derivaciones efectuadas por los profesionales del centro. Los diferentes conceptos de estudio 
fueron los siguientes: laboratorio, radiología, pruebas complementarias, interconsultas y prescripciones (recetas a cargo del CatSalut). Las tarifas utilizadas procedieron de los estudios de contabilidad analítica realizados en los centros o facturas de productos intermedios emitidas por los diferentes proveedores. Por tanto, el costo por paciente fue: $\mathrm{Cp}=$ (costo medio por visita $\mathrm{x}$ número de visitas [costos semifijos]) + (costos variables). Se efectuó un análisis bivariante con las pruebas ANOVA, chi cuadrado y correlación lineal de Pearson. Con la finalidad de conseguir una mejor adaptación a la normalidad de la distribución, en el análisis se realizó una transformación de la variable dependiente del costo de la asistencia a partir del logaritmo neperiano. El cálculo del poder explicativo se estableció en función del cociente de determinación $\left(R^{2}\right)$. Se utilizó el programa SPSSWIN versión 18.

El número de pacientes atendidos el 2008 fue de 227235. De estos, un 23,1 \% tenían 65 años a más; con una media de 75,9 años; un 60,1 \% fueron mujeres; el número de episodios fue de $6,3 \pm 5,2$, con 10,3 $\pm 12,2$ visitas realizadas/año. Las enfermedades más prevalentes fueron: vértigo/mareo $(26,5 \%)$, cefalea $(18,9 \%)$ y accidente cerebrovascular $(11,4 \%)$. La presencia de algún TN fue de 17,4 \% (IC95\%: 17,2-17,6 \%). Los pacientes con TN (en comparación con la población general) presentaron mayor promedio de edad $(77,6$ frente a 75,7 años; $p<0,001)$ y porcentaje de mujeres $(64,6$ frente a $59,1 \% ; p<0,001)$. El promedio del costo/unitario total fue de 2595 USD frente a 1736 USD, y el de medicamentos de 1629 USD frente a 1077 USD; respectivamente, $p<0,001$. El promedio de episodios/diagnósticos en los pacientes con TN fue de 8,8 frente a 5,9, p< 0,001. La edad muestra una baja correlación lineal con el costo de la asistencia $(r=0,136)$ y mayor con la influencia del número de episodios/diagnósticos $(r=0,498)$. La comorbilidad crónica en los pacientes con algún TN explica el $25,4 \%$ de la variabilidad del costo de la asistencia $\left(R^{2}\right.$ corregido por edad y sexo).

Los datos estiman que, en general, los TN son enfermedades de costo elevado. Es interesante destacar que en el ámbito de la AP se están haciendo muchos esfuerzos encaminados a tratar enfermedades o factores de riesgo de naturaleza cardiovascular, mientras que la tendencia es que se destinen más recursos al cuidado de estos pacientes, en especial a los de mayor edad. Por tanto, un escenario temporal a corto plazo en los equipos de AP, en la cuantificación de los costos (recursos en base a casuística) y adecuación flexible de la cartera de servicios (atención domiciliaria, ayuda a la dependencia, etc.), junto a la prevención de las enfermedades podría mejorar la capacidad de resolución y su enfoque social.
Estos resultados deben interpretarse con prudencia. Nuestra mayor limitación se presenta en la validez externa de los resultados, pues los centros analizados no necesariamente son representativos de toda la población, dado la organización, estilos de práctica clínica y características intrínsecas de los pacientes, pueden ocasionar un sesgo de contaminación. No obstante, los datos obtenidos en AP pueden ser una fuente de información importante para que las autoridades sanitarias adopten medidas apropiadas y costo-efectivas en este nivel asistencial, cada día más implicado con los TN. En conclusión, los pacientes que han demandado atención por algún TN presentan un elevado número de comorbilidades crónicas y costo anual. El número de comorbilidades crónicas presenta una mejor asociación que la edad en el uso de los recursos sanitarios, y se muestra como un sencillo predictor del costo de la asistencia en AP en situación de práctica clínica habitual.

\section{REFERENCIAS BIBLIOGRÁFICAS}

1. World Health Organization. The World Health Report 2002: reducing risk, promoting healthy life. Geneve: World Health Organization; 2002.

2. Boyd CM, Ritchie CS, Tipton EF, Studenski SA, Wieland D. From Bedside to Bench: summary from the American Geriatrics Society/National Institute on Aging Research Conference on Comorbidity and Multiple Morbidity in Older Adults. Aging Clin Exp Res. 2008;20:181-8.

3. John R, Kerby DS, Hennessy CH. Patterns and impact of comorbidity and multimorbidity among community-resident American Indian elders. Gerontologist. 2003;43:649-60.

4. Beguiristain JM, Mar J, Arrazola A. Costo de la enfermedad cerebrovascular aguda. Rev Neurol. 2005;40:406-11.

5. Sicras-Mainar A, Velasco-Velasco S. Patrón de uso de recursos y costos en pacientes que demandan atención por trastornos neurológicos en atención primaria. Rev Neurol. 2007;45:641-6.

6. O'Halloran J, Miller GC, Britt H. Defining chronic conditions for primary care with ICPC-2. Fam Pract. 2004;21:381-6.

\section{Correspondencia: Antoni Sicras-Mainar}

Dirección de Planificación. Badalona Serveis Assistencials SA; C. Gaietà Soler, 6-8 entlo. 08911 Badalona. Barcelona, España. Teléfono: (034) 935072684.

Correo electrónico: asicras@bsa.cat 